\title{
Juego de roles sociales, función simbólica y desarrollo de la personalidad en la edad preescolar
}

\author{
Social role play, symbolic function, and personality \\ development in preschool age
}

Claudia Ximena González-Moreno ${ }^{1}$

\section{RESUMEN}

Las formas maduras del juego de roles sociales posibilitan el desarrollo complejo de la función simbólica como aspecto indispensable para el desarrollo de la personalidad del niño en la edad preescolar. Este artículo presenta los resultados de una investigación realizada con un grupo de 20 niños en edad preescolar de la ciudad de Bogotá, Colombia a partir de una intervención en la que se usó el juego de roles sociales. Se identifican las características del juego de roles sociales y de la función simbólica en la medida que ambos se van complejizando a través de la orientación que ofrece el adulto. En general se aprecia una transformación tanto en el juego como en la función simbólica, sobre todo en la posibilidad de comprender al otro y de valorarlo. Se concluye que el desarrollo simbólico permite la formación de actitudes de abnegación en los niños. Por consiguiente, desarrolla la capacidad de ver el mundo a través de los ojos de los demás en interacciones comunicativas de reciprocidad emocional.

Palabras clave: Educación infantil. Desarrollo infantil. Educación preescolar. Juego infantil. Personalidad en la infancia.

\begin{abstract}
Mature forms of social role play enable complex development of symbolic function as an indispensable aspect for the development of the child's personality in preschool age. This article presents the results of an investigation carried out with a group of 20 preschool-age children from the city of Bogotá, Colombia, based on an intervention that used social role play. Characteristics of social role play and symbolic function are identified as both become more complex through the orientation offered by the adult. In general, a transformation can be seen both in the game and in the symbolic function, especially in the possibility of understanding and valuing the other. It is concluded that symbolic development allows the formation of self-denial attitudes in children. Consequently, she develops the ability to see the world through the eyes of others in communicative interactions of emotional reciprocity.
\end{abstract}

Keywords: Early childhood education. Child development. Preschool education. Children's play. Personality in childhood.

\footnotetext{
1 Doctora Interinstitucional en Educación, Universidad Iberoamericana de Puebla, México. Estudiante del Programa Postdoctoral de Investigación en Ciencias Sociales, Niñez y Juventud de la Universidad de Manizales, Colombia. ORCID: https:/orcid.org/0000-0001-8099-8605. E-mail: clauxigo@hotmail.com.
} 


\section{Introducción}

En la edad preescolar, la función simbólica se constituye en una formación psicológica fundamental que se complejiza cuando el niño participa en la actividad de juego de roles sociales (SOLOVIEVA; QUINTANAR, 2012; GONZÁLEZ-MORENO, 2015). De la organización de esta actividad depende la formación inicial de la personalidad al posibilitar en los niños tanto el desarrollo de mecanismos personales de la conducta (LEONTIEV, 1987) como de un repertorio emocional y simbólico amplio. En el juego de roles sociales los niños aprenden a ver la necesidad del otro, a ayudar mutuamente a los demás, a ser generosos, a desarrollar el espíritu de servicio, a trabajar cooperativamente y a dialogar entre unos y otros.

La investigación que se presenta en este artículo entiende el juego en primer lugar, como una actividad compartida social y cultural. En segundo lugar, como una actividad que propicia el desarrollo potencial en el niño, que se determina con la orientación y los tipos de apoyo que ofrece el adulto a través del uso de medios simbólicos en los niveles materializado (uso de objetos como sustitutos de otros), perceptivo (dibujos) y verbal (palabras). En tercer lugar, como una actividad que permite pensar cómo sería estar en el lugar de otra persona, de entender sus sentimientos y sus expectativas. En otras palabras, el juego posibilita que los niños vean el mundo desde una perspectiva más solidaria y compasiva.

En diversas investigaciones se ha encontrado que muchos niños no alcanzan el desarrollo de las formas maduras del juego (BREDIKYTE; HAKKARAINEN，2011; GONZÁLEZ-MORENO; SOLOVIEVA; QUINTANAR, 2014; GONZÁLEZ-MORENO, 2016). Las acciones de estos niños se caracterizan por ser aisladas y carecer de sentido emocional profundo por lo que es difícil que duren en el tiempo, además en ellos hay poca iniciativa y un pobre desenvolvimiento interaccional y comunicativo (GONZÁLEZ-MORENO, 2018). Los niños requieren que el juego avance hacia las formas maduras, lo cual se alcanza con la participación dialógica de todos los participantes: adulto y grupo 
de niños en la actividad de juego de roles sociales (HAKKARAINEN; BREDIKYTE, 2015; GONZÁLEZ-MORENO, 2016). Las formas maduras del juego posibilitan el desarrollo psicológico y de la personalidad infantil (BREDIKYTE; HAKKARAINEN, 2011). Asimismo, estas formas maduras del juego generan una necesidad social de comunicación entre un grupo de niños para que trabajen juntos; el rol (médico, enfermera, recepcionista, pacientes, pasajeros, chofer, ingeniero, ayudante, constructores, diseñadores, pintores, costureros, relojeros, trabajadores de gasolineras, panaderos) adquiere un sentido social; las acciones se relacionan con la representación de cada rol considerando el tema del juego; se aprende a comunicarse de manera efectiva, a autorregular el comportamiento y las emociones; se buscan soluciones conjuntas y colaborativas a problemas que surgen en el juego; se toman decisiones conjuntas de manera creativa; se desarrolla la habilidad de escucha activa, esfuerzo, ayuda mutua; se transforma el uso de medios simbólicos desde sus formas más sencillas hasta las más complejas (GONZÁLEZ-MORENO, 2015).

Por lo anterior, los objetivos de esta investigación fueron: 1. determinar cómo las formas maduras del juego de roles sociales promueven el desarrollo complejo de la función simbólica en los niveles materializado, perceptivo y verbal y 2. determinar cómo las formas maduras de juego de roles sociales y la función simbólica en el nivel complejo posibilitan el desarrollo de la personalidad en la edad preescolar.

\section{Método}

Esta investigación está orientada por el método experimental formativo propuesto por Vigotsky (1995), en el que se estudia la función simbólica durante su formación en el juego de roles sociales. Se usan diversos tipos de apoyo (conversación dialógica, preguntas de orientación, ejemplo, repetición de la instrucción) para influir positivamente sobre la función simbólica y el desarrollo de la personalidad en un grupo de niños.

Se consideran los planos del desarrollo intelectual: nivel simbólico materializado, nivel perceptivo y el nivel verbal. Estos niveles se relacionan 
con distintos planos de realización de la acción intelectual, tal y como se plantea en la teoría de formación de la acción por etapas (GALPERIN, 2000; SOLOVIEVA, 2014; TALIZINA, 2019).

La parte más importante en el proceso de formación de la función simbólica es la orientación, la cual dirige las acciones intelectuales y la solución de problemas que surgen, por lo que es necesario comprender cuál es la orientación que garantiza su ejecución (GALPERIN, 1976, 1995): dependiente o independiente, generalizada particular o general, completa o incompleta. La orientación puede modificar por completo la visión sobre el potencial del desarrollo del niño en lo que se refiere a la función simbólica en todas las etapas del juego de roles sociales (ver figura 1). Para poder crear la orientación es necesario un conocimiento amplio tanto del juego de roles sociales y sus etapas (juego de roles sociales con el uso de objetos, juego de roles sociales con sustitutos objetales, juego de roles sociales con uso de objetos y sustitutos objetales en varios tipos de situaciones, juego de roles sociales con incremento de iniciativa y propuesta de juegos nuevos, juego de roles sociales narrativo) como de la función simbólica y sus niveles de desarrollo (acciones materializadas, acciones perceptivas, acciones verbales) y de los cambios que van alcanzando los niños en sus actitudes hacia los demás (desarrollo de la personalidad) durante su participación en cada una de las etapas del juego con el uso del lenguaje en sus acciones. En cada etapa del juego de roles se le debe presentar al grupo de niños los objetos de su acción (objetos temáticos, objetos como sustitutos de otros, objetos imaginarios representados con acciones, dibujos y palabras), los tipos de relaciones que existen entre los objetos y lo que garantiza su aceptación como objetos de las acciones correspondientes a los roles que representan. 
Figura 1 - Tipos de apoyo en la orientación usados en la actividad de juego de roles sociales en sus diferentes etapas

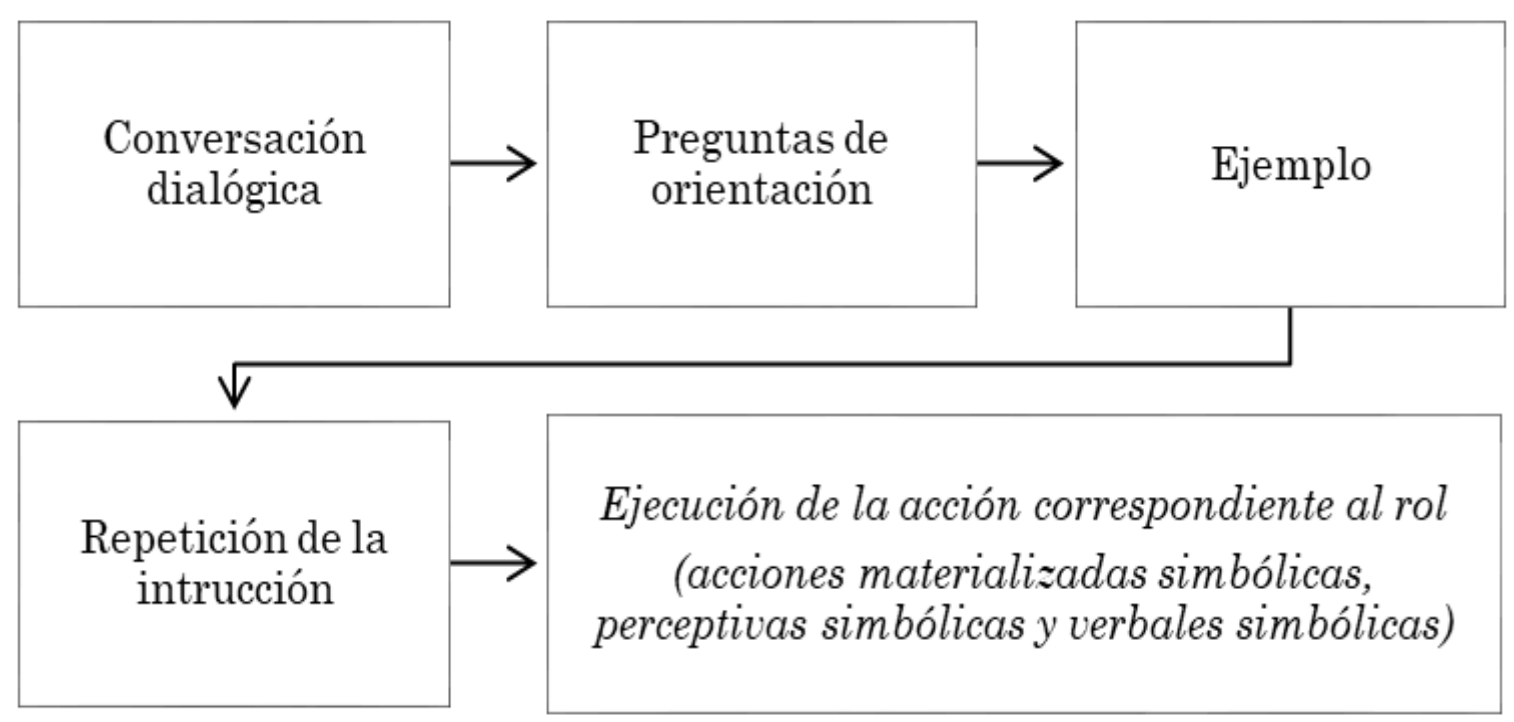

Fuente: Elaboración propia

\subsection{Participantes}

En el estudio participaron 20 niños de 5-6 años de último grado de preescolar de una institución educativa distrital de la ciudad de Bogotá, D. C, de los cuales 9 son niñas y 11 niños con una edad media de 5.2 años.

\subsection{Instrumentos y recolección de datos}

En la tabla 1 se presentan las etapas de juego diseñadas en esta investigación. En cada una de estas etapas se debe garantizar y despertar un interés o una necesidad particular en las interacciones dialógicas en los niños, así como considerar las orientaciones de los diversos planos de la acción (materializadas, perceptivas y verbales) que pueden ser útiles para las acciones que ellos representan a través de los roles reflexionando en la temática del juego. 
Tabla 1 - Etapas del juego de roles

\begin{tabular}{|c|c|c|c|}
\hline \multirow{2}{*}{$\begin{array}{l}\text { Etapa del juego } \\
\text { Etapa 1. Juego } \\
\text { de roles sociales } \\
\text { con el uso de } \\
\text { objetos }\end{array}$} & \multirow{2}{*}{\begin{tabular}{l}
\multicolumn{1}{c}{ Descripción } \\
Se utilizan objetos en la \\
representación de los roles.
\end{tabular}} & \multicolumn{2}{|c|}{ Criterios de análisis } \\
\hline & & Accede & $\begin{array}{l}\text { Realiza acciones con } \\
\text { objetos de acuerdo al rol } \\
\text { considerando la } \\
\text { orientación de la } \\
\text { investigadora }\end{array}$ \\
\hline & & Iniciativa & $\begin{array}{l}\text { Propone por su propia } \\
\text { iniciativa acciones con los } \\
\text { objetos de acuerdo al rol }\end{array}$ \\
\hline & & Reflexiona & $\begin{array}{l}\text { Está consciente de la } \\
\text { necesidad del uso de } \\
\text { objetos para participar en } \\
\text { su rol de manera activa y } \\
\text { los utiliza con frecuencia } \\
\text { caracterizando el } \\
\text { personaje que representa }\end{array}$ \\
\hline \multirow[t]{3}{*}{$\begin{array}{l}\text { Etapa } 2 . \text { Juego de } \\
\text { roles sociales con } \\
\text { sustitutos } \\
\text { objetales }\end{array}$} & \multirow[t]{3}{*}{$\begin{array}{l}\text { Se utilizan sustitutos de objetos en la } \\
\text { representación de los roles. }\end{array}$} & Accede & $\begin{array}{l}\text { Realiza acciones con } \\
\text { objetos como sustitutos de } \\
\text { acuerdo al rol considerando } \\
\text { la orientación de la } \\
\text { investigadora }\end{array}$ \\
\hline & & Iniciativa & $\begin{array}{l}\text { Propone por su propia } \\
\text { iniciativa acciones con los } \\
\text { sustitutos de objetos de } \\
\text { acuerdo al rol }\end{array}$ \\
\hline & & Reflexiona & $\begin{array}{l}\text { Está consciente de la } \\
\text { necesidad del uso de } \\
\text { objetos como sustitutos } \\
\text { para participar en su rol } \\
\text { de manera activa y los } \\
\text { utiliza con frecuencia } \\
\text { caracterizando el } \\
\text { personaje que representa }\end{array}$ \\
\hline \multirow[t]{2}{*}{$\begin{array}{l}\text { Etapa 3. Juego de } \\
\text { roles sociales con } \\
\text { uso de objetos y } \\
\text { sustitutos } \\
\text { objetales en } \\
\text { varios tipos de } \\
\text { situaciones }\end{array}$} & \multirow[t]{2}{*}{$\begin{array}{l}\text { Se utilizaron objetos y sustitutos de } \\
\text { objetos en la representación de los } \\
\text { roles. }\end{array}$} & Accede & $\begin{array}{l}\text { Realiza acciones con } \\
\text { objetos y con objetos como } \\
\text { sustitutos de acuerdo al } \\
\text { rol considerando la } \\
\text { orientación de la } \\
\text { investigadora }\end{array}$ \\
\hline & & Iniciativa & $\begin{array}{l}\text { Propone por su propia } \\
\text { iniciativa acciones con } \\
\text { objetos y con los } \\
\text { sustitutos de objetos de } \\
\text { acuerdo al rol }\end{array}$ \\
\hline
\end{tabular}




\begin{tabular}{|c|c|c|c|}
\hline & & Reflexiona & $\begin{array}{l}\text { Está consciente de la } \\
\text { necesidad del uso de } \\
\text { objetos y de objetos como } \\
\text { sustitutos para participar } \\
\text { en su rol de manera activa } \\
\text { y los utiliza con frecuencia } \\
\text { caracterizando el personaje } \\
\text { que representa }\end{array}$ \\
\hline \multirow[t]{3}{*}{$\begin{array}{l}\text { Etapa 4. Juego de } \\
\text { roles sociales con } \\
\text { incremento de } \\
\text { iniciativa y } \\
\text { propuesta de } \\
\text { juegos nuevos }\end{array}$} & \multirow[t]{3}{*}{$\begin{array}{l}\text { En esta etapa de juego los niños } \\
\text { proponen situaciones diversas de } \\
\text { juego y asumen roles. }\end{array}$} & Accede & $\begin{array}{l}\text { Utiliza expresiones } \\
\text { verbales de acuerdo a su } \\
\text { rol considerando la } \\
\text { orientación de la } \\
\text { investigadora }\end{array}$ \\
\hline & & Iniciativa & $\begin{array}{l}\text { Propone expresiones por } \\
\text { su propia iniciativa de } \\
\text { acuerdo al rol }\end{array}$ \\
\hline & & Reflexiona & $\begin{array}{l}\text { Está consciente de la } \\
\text { necesidad del uso de } \\
\text { expresiones características } \\
\text { del personaje que } \\
\text { representa } \\
\text { Se apropia de expresiones } \\
\text { características del rol con } \\
\text { frecuencia }\end{array}$ \\
\hline \multirow[t]{3}{*}{$\begin{array}{l}\text { Etapa } 5 \text {. Juego de } \\
\text { roles sociales } \\
\text { narrativo }\end{array}$} & \multirow{3}{*}{$\begin{array}{l}\text { En esta etapa de juego los niños } \\
\text { conjuntamente con el adulto } \\
\text { construyen historias en las que se } \\
\text { combinaban elementos de la } \\
\text { realidad y de la imaginación y } \\
\text { cada uno representa un rol. } \\
\text { Además, en esta etapa se observa } \\
\text { la elaboración compartida de } \\
\text { estructuras narrativas que } \\
\text { incluyen inicio de situaciones } \\
\text { imaginarias, desarrollo de una } \\
\text { situación que poco a poco se va } \\
\text { enriqueciendo, planteamiento y } \\
\text { solución del problema. } \\
\text { Los niños asumen roles } \\
\text { considerando el planteamiento } \\
\text { de la situación problema y la } \\
\text { logran resolver de manera } \\
\text { creativa y novedosa usando medios } \\
\text { simbólicos. }\end{array}$} & Accede & $\begin{array}{l}\text { El niño considera } \\
\text { algunas características } \\
\text { del rol que asume porque } \\
\text { la investigadora se lo } \\
\text { propone }\end{array}$ \\
\hline & & Iniciativa & $\begin{array}{l}\text { Destaca lo esencial (para } \\
\text { la acción), es decir, el } \\
\text { niño considera por su } \\
\text { iniciativa las } \\
\text { características específicas } \\
\text { de la acción de acuerdo al } \\
\text { rol que representa }\end{array}$ \\
\hline & & Reflexiona & $\begin{array}{l}\text { Clarifica de manera } \\
\text { independiente las } \\
\text { relaciones posibles del } \\
\text { problema a solucionar } \\
\text { representando un plan, la } \\
\text { cantidad de elementos y el } \\
\text { orden de las acciones a } \\
\text { realizar }\end{array}$ \\
\hline
\end{tabular}

Fuente: Elaboración propia

\subsection{Procedimiento}

El programa de intervención se realizó durante un año escolar, en total 
se hicieron 130 sesiones de juego. Este programa de intervención se diseñó considerando las aportaciones teóricas y metodológicas sobre el juego (ELKONIN, 1980; SOLOVIEVA Y QUINTANAR, 2012; HAKKARAINEN; BREDIKYTE, 2015).

El contenido de la intervención incluye los conocimientos de las temáticas de los juegos de roles que se proponen. El sistema de condiciones que orienta al niño al cumplimiento de la acción constituye la base de la orientación de la acción. Por esta razón, el contenido de la intervención establece la familiarización con las condiciones concretas de la acción. En un primer momento, la acción se realiza de la forma más desarrollada posible para que a los niños les quede clara la relación lógica del contenido concreto de la acción. Se identifican características relacionadas con la manera cómo los niños acceden a la actividad de juego de roles, aquí el adulto propone la actividad. Después se identifican características relacionadas con cómo empiezan a asumir el rol y cómo se apropian del personaje que están representando.

Como medios externos de apoyo se usan tarjetas de colores e imágenes, así como palabras y expresiones que le ayudan al niño a solucionar los problemas propuestos en los juegos de roles. De esta manera, se contribuye a modificar cualitativamente la manera como los niños asumen los roles de acuerdo a las temáticas. El juego considera las propuestas e intereses de los niños así como la necesidad de conseguir que sean juegos divertidos.

\subsection{Consideraciones éticas}

Este estudio se realizó dentro de las normas éticas que tienen su principio en la declaración de Helsinki de la Asociación Médica Mundial (2017), con la realización del consentimiento informado avalado por el comité ético del Doctorado Interinstitucional en Educación de la Universidad Iberoamericana de Puebla, el cual valoró la protección de los derechos fundamentales de los niños y de la institución educativa, de acuerdo con las exigencias legales. Asimismo, es necesario mencionar que los padres de los niños firmaron una carta de consentimiento informado en una reunión 
realizada en la institución educativa, y estos últimos manifestaron su asentimiento por medio de un dibujo que colorearon.

\section{Resultados}

El juego consistía en la interpretación de los roles, sin embargo, esta interpretación no surgió desde el inicio. La investigadora propuso los roles, después los niños empezaron a denominarlos, aunque los roles no determinaban su actitud en el juego. Luego los niños iniciaron con la representación de los roles esporádicamente (realizaban algunas acciones y abandonaban el rol) y finalmente asumieron la caracterización del personaje enriqueciendo sus acciones. Se observó que los niños usaban diversos medios representativos expresivos como el cambio en la entonación de la voz, los gestos, las acciones y actitudes especiales con el rol que asumían. De la misma manera, los niños utilizaban un objeto como sustituto de otro atribuyéndole múltiples funciones en el juego de acuerdo a las necesidades que se generaban de manera grupal. También expresaban las propias ideas para crear situaciones imaginarias e historias que lograban representar por medio de los roles. Al inicio el sentido del juego se centró en las acciones con los objetos y con los sustitutos de objetos, después el sentido se reflejó en las relaciones con los otros niños y luego el sentido se fundamentó en las relaciones características del rol que se representaba y se relacionó con los otros roles de manera creativa e imaginativa considerando las reglas.

Los niños necesitaron que la investigadora estableciera relaciones específicas entre los roles y sus acciones de manera verbal y a través de su representación, lo que los cautivó y posibilitó la ampliación de los argumentos al incrementar y complejizar las acciones. De la misma manera, los niños comprendieron el significado de sus acciones en el juego lo que hizo que aparecieran nuevas acciones. Las acciones con los objetos concretos se transformaron en acciones simbólicas e imaginativas y después en acciones que hacían parte de los roles.

Los niños cambiaban de actitud con el rol que representaban considerando la comprensión y el sentido que alcanzaban de esta 
interpretación a medida que transcurrían las sesiones interventivas. Como medio de significación de las acciones los niños usaban el lenguaje. Al inicio, los niños usaron el lenguaje como medio de comunicación con los otros roles, después lo usaron como medio de regulación del comportamiento (hablaban para planear acciones o mientras realizaban acciones diversas con sustitutos de objetos o con objetos imaginarios). La acción se iba complejizando a medida que los niños se hacían conscientes de la identificación de las acciones de los roles de los demás y de las de su propio rol, lo que les permitió separarse del otro y ejecutar de la mejor manera posible sus acciones para caracterizar el personaje que estaban representando. El hecho de adoptar la postura de un personaje y asumir un rol determinado sintiendo lo que hacía y decía llevó al niño a comportarse por encima de su desarrollo, lo que permitió encontrar la zona de desarrollo próximo.

Además, al asumir roles los niños tenían la posibilidad de ponerse en el lugar del otro lo que permitía aprender del otro rol y a través del otro rol. Cuando un rol estaba aprendiendo a través de otro rol se lograba identificar con él y con sus intenciones. Esto facilitaba la creación de nuevos medios simbólicos que iban desde los más sencillos (medios materializados simbólicos) hasta los más complejos (medios verbales simbólicos) en la interacción social, lo que pasó a ser una fuente cultural de regulación del comportamiento (ver tabla 2). 
Tabla 2 - Complejización de la función simbólica en las etapas del juego de roles sociales

\begin{tabular}{|c|c|c|c|}
\hline \multirow{2}{*}{$\begin{array}{l}\text { Etapa del } \\
\text { juego }\end{array}$} & \multicolumn{3}{|c|}{ Nivel de desarrollo de la función simbólica } \\
\hline & Acciones materializadas & $\begin{array}{l}\text { Acciones } \\
\text { perceptivas }\end{array}$ & Acciones verbales \\
\hline $\begin{array}{l}\text { Juego de roles } \\
\text { sociales con el } \\
\text { uso de objetos }\end{array}$ & $\begin{array}{l}\text { El niño requiere del uso de } \\
\text { juguetes temáticos y del uso } \\
\text { de objetos característicos del } \\
\text { rol que representa para } \\
\text { expresar las acciones } \\
\text { correspondientes. }\end{array}$ & $\begin{array}{l}\text { El niño realiza las } \\
\text { mismas acciones que el } \\
\text { adulto u otros niños al } \\
\text { observar medios } \\
\text { simbólicos perceptivos. }\end{array}$ & $\begin{array}{l}\text { El niño utiliza } \\
\text { algunas expresiones } \\
\text { verbales de acuerdo al } \\
\text { rol que representa. }\end{array}$ \\
\hline $\begin{array}{l}\text { Juego de roles } \\
\text { sociales con } \\
\text { sustitutos } \\
\text { objétales }\end{array}$ & $\begin{array}{l}\text { 1.El niño imita el uso de } \\
\text { objetos como sustitutos de } \\
\text { otros de acuerdo a lo que } \\
\text { observa que propone el } \\
\text { adulto. } \\
\text { 2.Uso inestable o } \\
\text { inconsistente de un objeto } \\
\text { como sustituto de otro. }\end{array}$ & $\begin{array}{l}\text { El niño utiliza de } \\
\text { manera inestable o } \\
\text { inconsistente medios } \\
\text { simbólicos en el plano } \\
\text { gráfico. }\end{array}$ & $\begin{array}{l}\text { 1.El niño muestra } \\
\text { interés por las } \\
\text { expresiones verbales } \\
\text { que usan otros niños o } \\
\text { el adulto en sus roles. } \\
\text { 2.El niño utiliza de } \\
\text { manera inestable o } \\
\text { inconsistente medios } \\
\text { simbólicos verbales. } \\
\text { en los roles. }\end{array}$ \\
\hline $\begin{array}{l}\text { Juego de roles } \\
\text { sociales con } \\
\text { uso de objetos } \\
\text { y sustitutos } \\
\text { objétales en } \\
\text { varios tipos de } \\
\text { situaciones }\end{array}$ & $\begin{array}{l}\text { 1.El niño le da el nombre al } \\
\text { objeto como sustituto de otro } \\
\text { antes de realizar una acción } \\
\text { de sustitución con él. } \\
\text { 2.Surgen secuencias de } \\
\text { acciones con los objetos como } \\
\text { sustitutos de otros. }\end{array}$ & $\begin{array}{l}\text { Propuesta } \\
\text { independiente del uso } \\
\text { de medios simbólicos } \\
\text { en el plano gráfico. }\end{array}$ & $\begin{array}{l}\text { Posibilidad de crear } \\
\text { situaciones } \\
\text { imaginarias a través } \\
\text { de palabras que } \\
\text { enriquecen las } \\
\text { acciones. }\end{array}$ \\
\hline $\begin{array}{l}\text { Juego de roles } \\
\text { sociales con } \\
\text { incremento de } \\
\text { iniciativa y } \\
\text { propuesta de } \\
\text { juegos nuevos }\end{array}$ & $\begin{array}{l}\text { El niño juega con objetos } \\
\text { imaginarios. }\end{array}$ & $\begin{array}{l}\text { El niño usa los medios } \\
\text { simbólicos en el plano } \\
\text { gráfico para regular el } \\
\text { comportamiento de los } \\
\text { otros roles y su propio } \\
\text { rol. }\end{array}$ & $\begin{array}{l}\text { Surge la habilidad de } \\
\text { considerar la } \\
\text { perspectiva del otro. }\end{array}$ \\
\hline $\begin{array}{l}\text { Juego de roles } \\
\text { sociales } \\
\text { narrativo }\end{array}$ & $\begin{array}{l}\text { Surgen secuencias de } \\
\text { acciones desplegadas en el } \\
\text { tiempo con objetos } \\
\text { imaginarios. }\end{array}$ & $\begin{array}{l}\text { Los niños proponen el } \\
\text { uso de símbolos } \\
\text { gráficos (íconos) por su } \\
\text { propia iniciativa y les } \\
\text { atribuyen significado } \\
\text { emocional. }\end{array}$ & $\begin{array}{l}\text { 1. Posibilidad de } \\
\text { representar diferentes } \\
\text { roles en diferentes } \\
\text { escenarios } \\
\text { imaginarios. } \\
2 . \text { El niño crea } \\
\text { personajes } \\
\text { imaginarios en el } \\
\text { juego y logra } \\
\text { continuar el vínculo } \\
\text { comunicativo con ellos } \\
\text { y con los demás roles. }\end{array}$ \\
\hline
\end{tabular}

Fuente: Elaboración propia. 
Así, por ejemplo, en el juego "la búsqueda del tesoro", los niños utilizaron un mapa para planear sus acciones. En esta sesión de juego los niños crearon una situación imaginaria con diversos objetos como sustitutos de otros (una caja de cartón grande era el barco, pedazos de papel verde eran los árboles, unas fichas redondas eran las rocas, un pedazo de papel azul era un lago, un tubo de plástico era una catapulta, unos cubos de cartón eran el castillo), también usaron representaciones esquematizadas (flechas para señalar la orientación de las acciones y una cruz que indicaba el lugar en el que se encontraba el tesoro).

En la figura 2 se presenta un ejemplo de ejecución en el plano perceptivo. Los niños dibujaron un barco con una bandera para indicar que desde ese lugar salían a cumplir con su misión "encontrar el tesoro", después dibujaron unos árboles para mostrar que tenían que atravesar un gran bosque lleno de animales peligrosos, luego tenían que dirigirse a una montaña rocosa en donde había una pista secreta que les mostraría como atravesar un lago para llegar a donde estaba la catapulta y derribar una muralla encantada porque debajo estaba el tesoro. Ellos tenían que llevar el tesoro de regreso al castillo del rey. En el castillo dibujaron un corazón para mostrar que cuando cumplieran la misión el rey iba a están muy alegre.

Figura 2 - Relación juego de roles sociales, función simbólica y personalidad

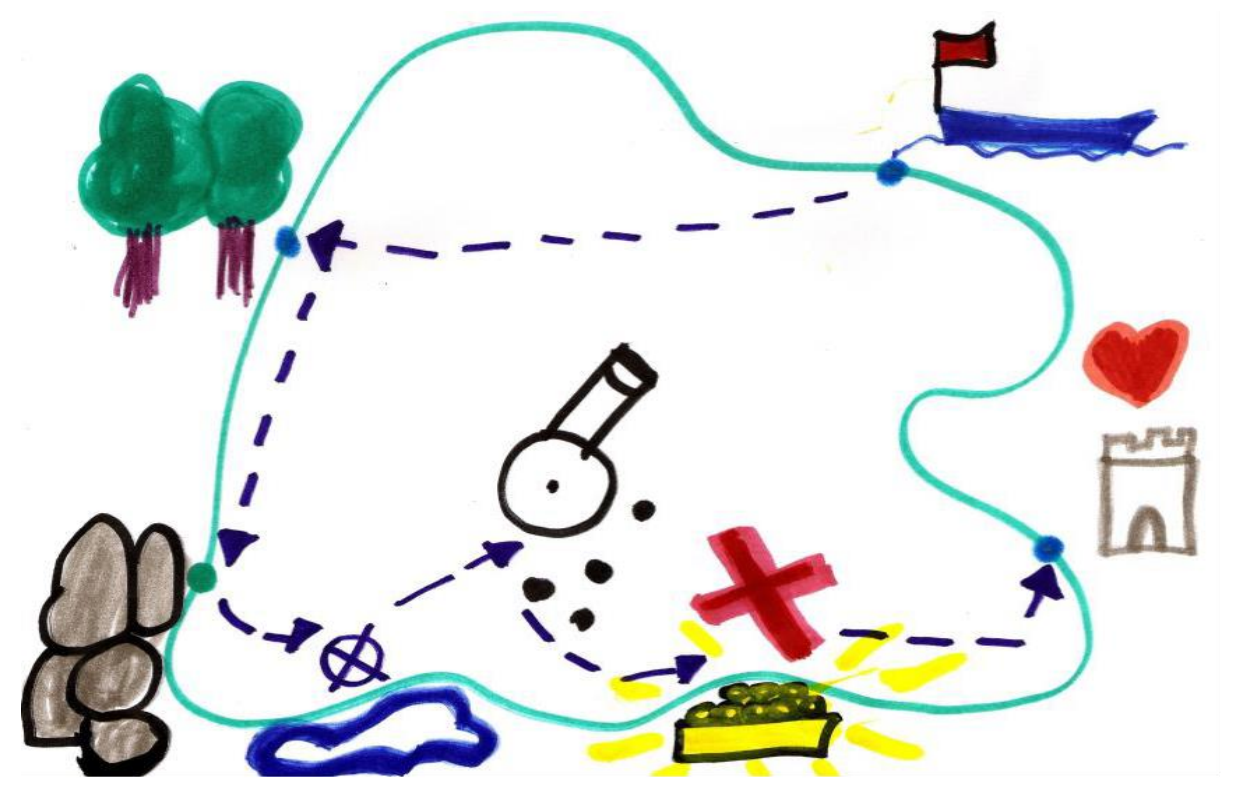

Mapa que señala las acciones en el juego de acuerdo a signos y esquemas para encontrar el tesoro. Dibujo grupal

Fuente: Elaboración propia 
En la figura 3 se presenta otro ejemplo de planificación de acciones y de creación de símbolos para resolver una situación problema en el juego "atrapados en un bosque profundo”. En esta sesión de juego los niños recrearon una situación imaginaria en la que debían descubrir qué había en el bosque profundo y oscuro para salir y regresar victoriosos a casa. Los niños señalizaron con una " $\mathrm{x}$ " el lugar en donde se encontraban. Después hicieron flechas para mostrar que tenían que atravesar una gran montaña helada para encontrar tres flores mágicas con poderes especiales que los llevarían a localizar los escudos del valor representados con color verde y con un corazón, lo cual les permitiría defenderse del feroz dragón y de esta manera podrían encontrar luces de corazón que les mostraría el camino a casa. En esta sesión de juego los niños comunicaron creativamente tanto de manera verbal como icónica su manera personal de comprender la situación imaginaria a través de los medios simbólicos.

Figura 3 - Ejemplo de planificación de acciones en el juego "atrapados en un bosque profundo"

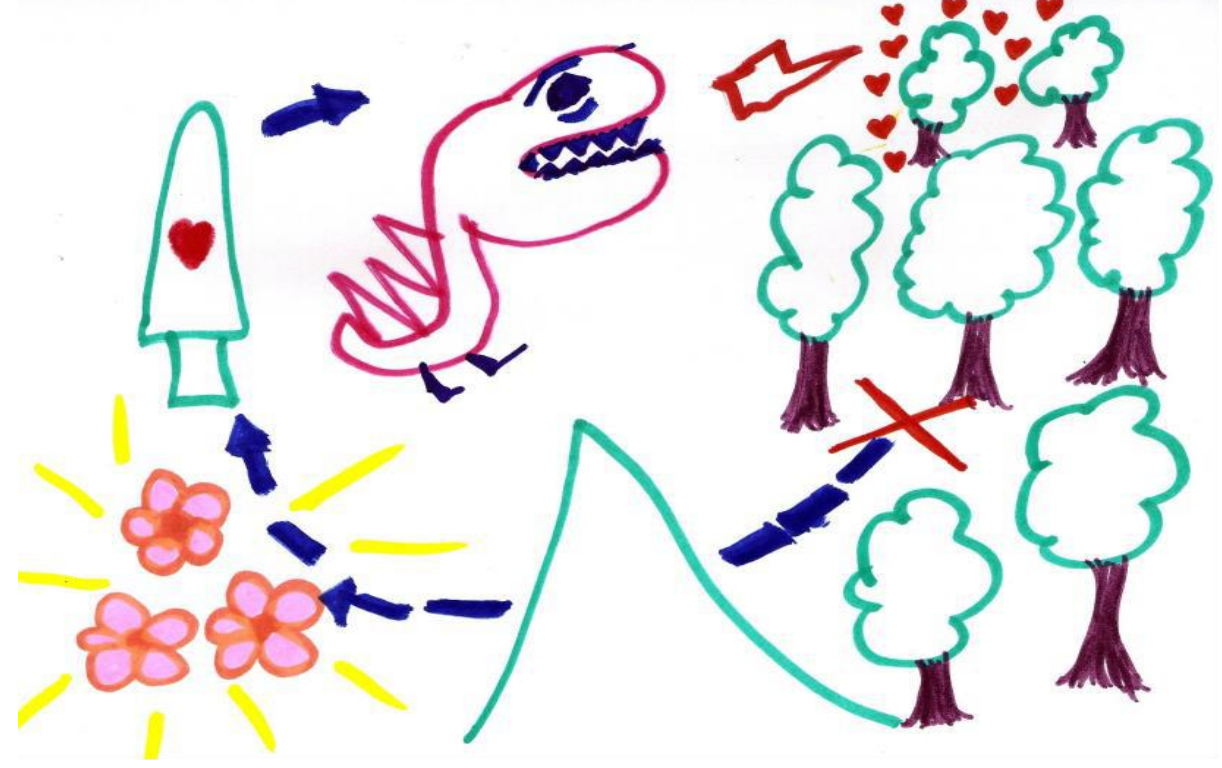

Mapa de orientación de acciones para resolver la situación problema imaginaria "descubrir qué había en el bosque profundo y oscuro". Dibujo grupal

Fuente: Elaboración propia

En la figura 4 se presenta un ejemplo de conversación dialógica creativa durante la ejecución del juego "Pinocho no quiere sopa". En esta sesión de juego se observa que los niños juegan con objetos imaginarios, crean eventos imaginarios 
nuevos de acuerdo a las necesidades de la actividad y muestran iniciativa para resolver problemas encontrando una solución que es aceptada por los otros.

Figura 4 - Ejemplo de conversación dialógica creativa durante la ejecución del juego "Pinocho no quiere sopa"

Niño (AI): ayuda.

Niño (JD): ¿quién anda por ahí?

Niño (JG): escucho una vocecita por aquí.

Niño (DP): yo también escucho una vocecita, pero no veo a nadie.

Investigadora: alguien está halando mi vestido.

¿Quién es?

Niña (AD): y el mío también.

Niño (AI): por favor no me vayan a pisar.

Investigadora: yo tengo una lupa para ver por aquí.

Niño (RM): yo también tengo una lupa.

Niño (JL): y yo.

Niño (RM): veo a un niñito.

Niño (JL): ese niñito es muy chiquito.

Investigadora: ¿cómo te llamas?

Niño (AI): soy Pinocho.

Niño (HS): $\iota y$ dónde está tu casita?

Niño (AI): yo vivo aquí.

Niño (JL): se me cayó mi lupa. Ya no te veo. ¿Por

dónde estás?

Niño (AI): yo sigo aquí.

Niña (MC): ¿qué te pasa?

Niño (AI): tú tienes forma de pastelito.

Niña (MV): yo no soy un pastelito.

Niño (CI): ¿por qué estás triste?

Niño (AI): ¿tú tienes forma de juguito?

Niño (CI): yo no soy juguito.

Niño (AI): es que me duele mucho la barriguita porque desde hace varios días no como nada.

Niña (MV): ¿qué te gustaría comer?

Niño (AI): a mí me gusta el juguito de las flores para preparar pastelitos y para tomar juguito, pero ya no hay ni una sola gota en ninguna de las flores.

Investigadora: yo veo un pozo de agua muy cerca, te voy a traer un poquito de agua.

Niña (EC): yo te acompaño y le traemos toda el agua que podamos en esta botellita (movimiento de la mano especial-objeto imaginario).

Niño (AI): mi barriguita me duele mucho auch.

Niño (CI): ya casi te traemos comidita.

Niño (AI): allá arriba veo pastelitos de caramelo.

Niño (AL): ¿dónde ves pastelitos de caramelo?

Niño (AI): allá arriba (señala con su mano)

Niño (AL): yo te puedo traer uno.

Niño (AI): yo quiero ir.

Niño (AL): vamos juntos.

Niño (SB): yo también quiero ir.

Niño (RM): yo también.

Niña (EC): aquí te trajimos un poquito de agua, toma un poquito.
Niño (AI): (hace gestos imaginando que está

tomando agua)

Niño (AL): ahora vamos por los pastelitos de caramelo que están allá arriba.

Niño (AI): me sigue doliendo mucho mi barriguita.

Niña (MV): yo soy doctora y te puedo revisar la

barriguita con este aparato especial (objeto imaginario)

Niño (AI): auch, auch duele mucho.

Niña (MV): ¿por aquí te duele?

Niño (AI): sí.

Niña (MV): ¿y por aquí te duele?

Niño (AI): si me duele.

Niña (MV): toma más agüita.

Niño (AI): sí.

Niña (MV): ahora toma esta pastillita (objeto imaginario)

Niño (AI): gracias, ya me siento mejor.

Niño (AL): ahora si podemos ir por los pastelitos

de caramelo.

Niño (AI): sí.

Investigadora: pero tenemos que atravesar una laguna.

Niño (SB): ¿cómo vamos a hacer para atravesar esa laguna?

Niño (HS): ya sé, digamos que había lagartijas en la laguna.

Investigadora: entonces las lagartijas nos ayudaban a cruzar la laguna.

Niño (JG): pero las lagartijas son bravas.

Niña (EC): pero digamos que les gustaba la música.

Niña (AD): y si tocamos flautas mágicas, yo tengo una aquí (flauta imaginaria)

Niña (KC): yo la puedo escuchar, suena bonito.

Niña (MC): entonces si tocas esa flauta mágica las lagartijas ya no van a estar más bravas.

Investigadora: $\mathrm{y}$ entonces podremos atravesar la laguna.

Niña (AD): ya estoy tocando mi flauta mágica.

Investigadora: entonces vamos por aquí

Niño (CI): Pinochito yo te puedo ayudar a pasar.

Niño (AL): llegamos.

Niño (CI): vamos por los pastelitos de caramelo.

Niño (DP): se los comió la lagartija gigante

(animal imaginario).

Niño (AI): y ahora.

Niño (DP): miren se acercan muchas moscas gigantes.

Niño (CI): y parece que están muy enojadas.

Niño (DP): es que los pastelitos de caramelo eran la comida favorita de las moscas. 
Investigadora: corran, podríamos entrar a esta cueva secreta (lugar imaginario construido con mantas de colores)

Niño (DP): ya se fueron las moscas gigantes. Investigadora: ya se está haciendo de noche. Niña (MV): yo tengo mucha hambre.

Niña (EC): y yo.

Niña $(\mathrm{AD})$ : podriamos preparar algo de comida para todos.

Niña (EC): yo tengo mi botellita con mucha agua (acciones imaginarias).

Niño (HS): y si preparamos una sopita calientita es que también está haciendo mucho frío.

Niño (RM): sí.

Niño (DP): todo está muy oscuro, necesitamos algo de luz.

Investigadora: digamos que había luciérnagas.

Niña (MV): ¿luciérnagas?

Investigadora: las luciérnagas son animalitos que tienen en su colita luz y pueden alumbrar en la oscuridad.

Niña: si había muchas luciérnagas que nos alumbraban mucho por aquí.

Niño (CI): podemos hace sopita con agua y con hojitas (acciones imaginarias).

Niña (EC): digamos que esta botellita se convertía en una olla gigante con ojitos.

Niña $(A D)$ : y podríamos poner unas piedritas de colores para que la ollita no se caiga (fichas de colores)

Investigadora: y digamos que si todos soplamos podemos encender el fuego para que se caliente la sopa.

Niño (AI): a mí no me gusta la sopa, solo me gustan los pastelitos.

Niña (MV): pero ya no hay pastelitos porque se los comieron toditos las lagartijas.

Niño (AI): yo no quiero sopa.

Niña (MC): pero esta sopita es rica.

Niño (AI): pero es que a mí no me gusta la sopa.

Niña (MV): pero y si tomas un poquito, te vas a

sentir mejor y ya no te va a doler tu barriguita.

Niño (AI): está bien, pero solo tomo un poquito de sopa, solo un poquito.

Investigadora: ya está lista la sopa.

Niña (MV): voy a servirte un poquito de sopa en esta tacita (objeto imaginario)

Niña (EC): tomate tu sopita.

Niño (AI): está muy caliente.
Niño (HS): yo te la puedo ayudar a enfriar con mis súper soplidos.

Niño (EC): ya está lista tu sopita.

Niño (AI): sigue muy caliente.

Niño (SB): yo también tengo súper soplidos y puedo ayudar a soplar tu sopita para que se enfríe.

Niño (CI): yo quiero tocar tu tacita (acciones imaginarias). Ya no está caliente, está tibiecita puedes tomarte tu sopita Pinochito.

Niño (AI): (gestos imaginarios que muestran que se está tomando la sopa).

Niña (MV): toma más sopita, te hará bien para tu barriguita.

Niña (EC): mira todos nos tomamos nuestra sopita en nuestras tacitas (acciones imaginarias).

Investigadora: esta sopita está muy rica.

Niña (EC): le vamos a poner un ingrediente especial a tu sopita.

Niña (MV): Pinochito prueba tu sopita, está muy rica.

Niño (AI): es que me duele mucho mi barriguita.

Niña (MV): voy a revisarte tu barriguita. ¿Te

duele?

Niño (AI): auch.

Niña (EC): voy a ponerte mi mano aquí para que no te duela más.

Niña (AD): yo te puedo dar un masaje en tu barriguita.

Niña (MV): ahora ¿cómo te sientes?

Niño (AI): ya me siento mucho mejor.

Niña (EC): ahora tomate tu sopita.

Niño (HS): ya todos nos tomamos toda la sopita,

solo faltas tú.

Niño (AI): está bien.

Investigadora: ahora es muy tarde todos podemos quedarnos a dormir aquí esta noche.

Niños: sí. (Todos realizan acciones imaginarias como si estuvieran dormidos)

Investigadora: ahora que es de día vamos a regresarnos en el tren encantado al jardín de Pinocho para regresarlo a su casa.

Niños: suban por aquí (acciones imaginarias) Niño (HS): llegamos.

Niño (DP): podemos construirle a Pinochito un columpio cerca de su casita.

Niña (MV): y le dejamos la ollita para que pueda preparar su comida y no le vuelva a doler su barriguita.

Fuente: Elaboración propia

Cuando los niños comprendieron las intenciones del otro no solo apoyaban sus acciones por medio de gestos y palabras sino que también trataban de explicar la situación a través de medios simbólicos perceptivos. En la figura 5 se presentan algunos ejemplos de símbolos creados en el juego "Pinocho no quiere sopa". 
Figura 5 - Ejemplo de símbolos creados en el juego "Pinocho no quiere sopa"

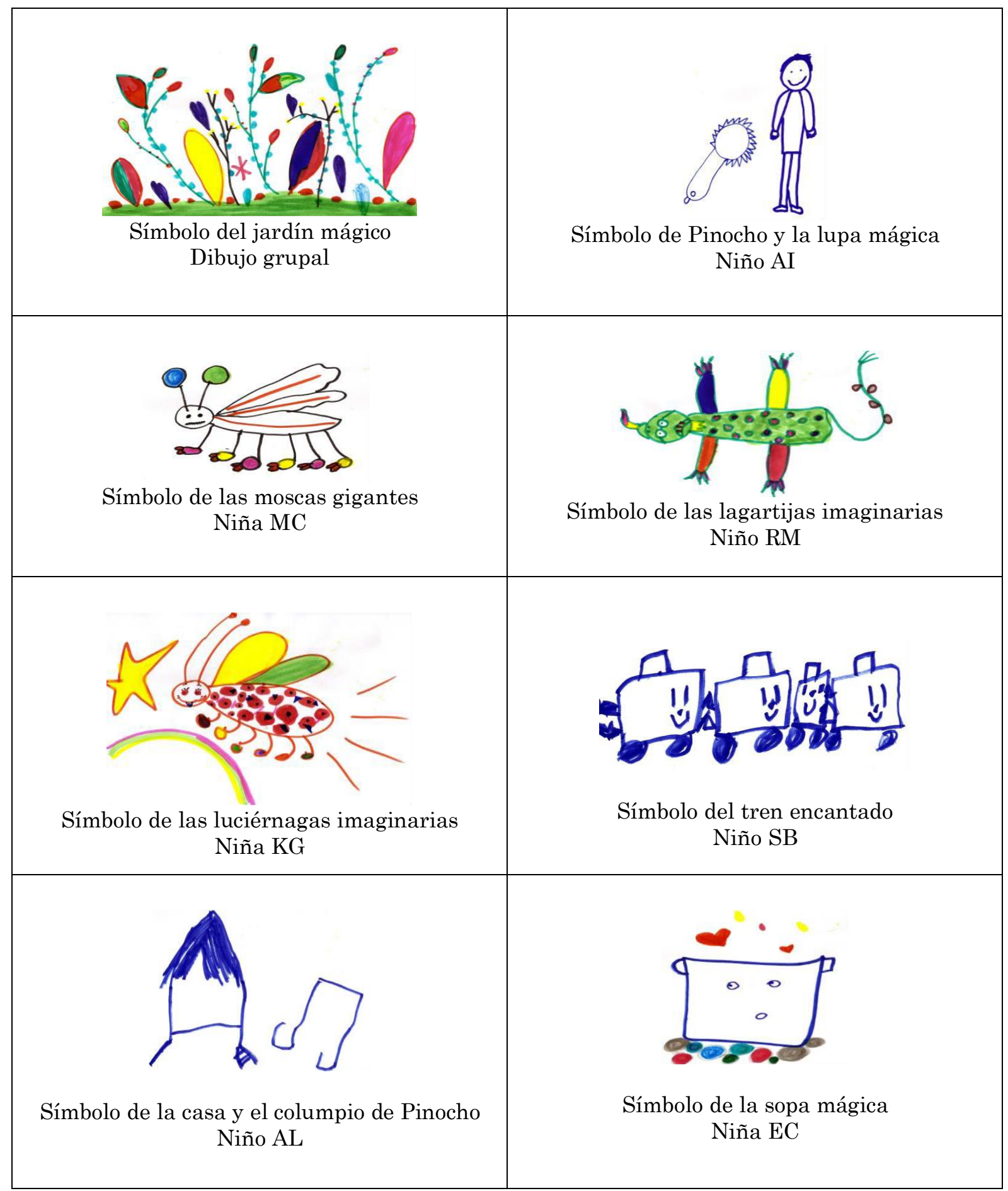

Fuente: Elaboración propia

Durante la participación de los niños en el juego se observaron transformaciones tanto en sus acciones como en sus interaciones sociales, las reorientaban hacia un nuevo contenido en la etapa de juego de roles narrativo (el 
bienestar del otro), se formaron nuevos motivos de conducta (compasión hacia el otro y actitudes de abnegación) y las interrelaciones con las personas modificaron su actitud hacia lo circundante y hacia sí mismos. También se observó que al inicio los niños percibían de los objetos y las situaciones lo que para ellos tenía sentido y respondía a sus necesidades, después ellos mismos transformaron sus propias acciones considerando las necesidades de los demás (ver tabla 3).

Tabla 3 - Desarrollo de la personalidad en las etapas de juego de roles sociales

\begin{tabular}{|c|c|c|c|c|c|}
\hline \multirow{2}{*}{$\begin{array}{l}\text { Etapa del } \\
\text { juego }\end{array}$} & \multicolumn{5}{|c|}{ Desarrollo de la personalidad } \\
\hline & $\begin{array}{l}\text { Desarrollo } \\
\text { emocional }\end{array}$ & $\begin{array}{c}\text { Desarrollo } \\
\text { moral }\end{array}$ & $\begin{array}{l}\text { Desarrollo de la } \\
\text { voluntad }\end{array}$ & $\begin{array}{l}\text { Nivel de } \\
\text { regulación } \\
\text { conductual }\end{array}$ & $\begin{array}{c}\text { Esfera } \\
\text { motivacional }\end{array}$ \\
\hline $\begin{array}{l}\text { Juego de roles } \\
\text { sociales con el } \\
\text { uso de objetos }\end{array}$ & $\begin{array}{l}\text { El uso de objetos } \\
\text { adquiere un } \\
\text { caracter afectivo } \\
\text { en la } \\
\text { representación } \\
\text { de los roles e } \\
\text { impulsa sus } \\
\text { acciones en las } \\
\text { representación } \\
\text { de los roles. }\end{array}$ & $\begin{array}{l}\text { El niño obedese } \\
\text { a sus deseos, } \\
\text { observa y rompe } \\
\text { la regla del } \\
\text { juego. }\end{array}$ & $\begin{array}{l}\text { Tendencia a } \\
\text { actuar } \\
\text { impulsivamente, } \\
\text { el niño inicia la } \\
\text { representación de } \\
\text { un rol y no logra } \\
\text { finalizar. }\end{array}$ & $\begin{array}{c}\text { Inicia la } \\
\text { consciencia } \\
\text { de sí como } \\
\text { sujeto de la } \\
\text { acción en la } \\
\text { interacción } \\
\text { con objetos } \\
\text { temáticos en } \\
\text { las } \\
\text { interacciones } \\
\text { sociales del } \\
\text { juego. }\end{array}$ & $\begin{array}{l}\text { Los motivos } \\
\text { externos } \\
\text { orientan la } \\
\text { acción del niño } \\
\text { considerando } \\
\text { primcipalment } \\
\text { e el uso de los } \\
\text { objetos. }\end{array}$ \\
\hline $\begin{array}{l}\text { Juego de roles } \\
\text { sociales con } \\
\text { sustitutos } \\
\text { objétales }\end{array}$ & $\begin{array}{l}\text { El uso de objetos } \\
\text { como sustitutos } \\
\text { de otros } \\
\text { adquiere un } \\
\text { caracter afectivo } \\
\text { en la } \\
\text { representación } \\
\text { de los roles e } \\
\text { impulsa sus } \\
\text { acciones. }\end{array}$ & $\begin{array}{c}\text { Surge la } \\
\text { contradicción } \\
\text { entre el quiero y } \\
\text { el debo, lo que } \\
\text { pone al niño } \\
\text { ante la } \\
\text { necesidad de } \\
\text { elección. }\end{array}$ & $\begin{array}{c}\text { El niño no logra } \\
\text { seguir las reglas } \\
\text { del juego } \\
\text { completamente. } \\
\text { El niño actua de } \\
\text { acuerdo a las } \\
\text { circunstancias que } \\
\text { actúan sobre él. }\end{array}$ & $\begin{array}{c}\text { Inicia la } \\
\text { consciencia } \\
\text { de sí como } \\
\text { sujeto de la } \\
\text { acción en la } \\
\text { interacción } \\
\text { con objetos } \\
\text { como } \\
\text { sustitutos de } \\
\text { otros en las } \\
\text { interacciones } \\
\text { sociales del } \\
\text { juego. }\end{array}$ & $\begin{array}{l}\text { Los motivos } \\
\text { externos } \\
\text { orientan la } \\
\text { acción del niño } \\
\text { considerando } \\
\text { principalment } \\
\text { e el uso de } \\
\text { objetos como } \\
\text { sustitutos de } \\
\text { otros. }\end{array}$ \\
\hline
\end{tabular}




\begin{tabular}{lcc}
\hline Juego de roles & El uso de objetos & En la \\
sociales con & y de objetos & interacción con \\
uso de objetos & como sustitutos & el otro surgen \\
y sustitutos & de otros & ideas y \\
objétales en & adquiere un & sentimientos \\
varios tipos de & caracter afectivo & morales. \\
situaciones & en la & \\
& representación \\
& de los roles e \\
& impulsa el \\
& desarrollo de \\
& nuevas acciones.
\end{tabular}

El niño empieza a renunciar a lo que más quiere, empieza a guiarse por aspiraciones $\mathrm{y}$ deseos estables.
Se fortalece la consciencia de sí como sujeto de la acción en la interacción con objetos y con objetos como sustitutos de otros en las interacciones sociales del juego.

\begin{tabular}{lc}
\hline $\begin{array}{l}\text { Juego de roles } \\
\text { sociales con }\end{array}$ & $\begin{array}{c}\text { El niño reconoce } \\
\text { que existen }\end{array}$ \\
incremento de & vivencias \\
iniciativa y & afectivas en el \\
propuesta de & juego de roles, lo \\
juegos nuevos & cuan se \\
& manifiesta a \\
& través de \\
& expresiones \\
& verbales.
\end{tabular}

Inicia la
formación de la
necesidad de
comunicación
interactiva y
dialógica con
otros roles, de
recibir su
atención y apoyo.
Los sentimientos
morales poseen
una fuerza
mpulsiva mayor.

Posibilidad de plantear objetivos conjuntos con los otros participantes del juego. atención y apoyo.

Los sentimientos una fuerza

impulsiva mayor.
Formación de intenciones de participar activamente a nivel de las interacciones sociales.
Inicia el proceso de formación de objetivos motivadores, coloreados afectivamente.
Surgen motivaciones en el plano interno de las acciones:

vínculo con los otros.

\begin{tabular}{lc}
\hline Juego de roles & Se fortalecen las \\
sociales & experiencias \\
narrativo & afectivas que se \\
& generan en el \\
& juego de roles.
\end{tabular}

\section{Inicio de relación} armónica entre procesos cognoscitivos, afectivos y voluntarios.

Se fortalecen las interacciones comunicativas con los otros roles y la búsqueda de atención y apoyo de los demás, así como la necesidad de atender a necesidades de los demás y de apoyarlos.

El niño puede guiarse por aspiraciones $\mathrm{y}$ deseos estables ligados con la asimilación de normas sociales.

$\begin{array}{ccc}\begin{array}{c}\text { Posibilidad de } \\ \text { dirigir el propio } \\ \text { comportamiento. }\end{array} & \begin{array}{c}\text { Orientación } \\ \text { consciente de } \\ \text { la propia } \\ \text { conducta en } \\ \text { el juego. }\end{array} & \begin{array}{c}\text { Formación de } \\ \text { actitudes de } \\ \text { abnegación. }\end{array} \\ & \end{array}$

Fuente: Elaboración propia. 


\section{Discusión}

Al analizar cada etapa del juego de roles sociales se encontró que el uso de los objetos en el primer nivel fue necesario para la organización de las acciones y su complejización desde la acción aislada, pasando por la presencia de dos acciones a la cadena de acciones con sustitutos objetales y objetos imaginarios. Esto generó la posibilidad de solucionar situaciones conflictivas a partir de varias opciones de actuación y de la reflexión acerca del estado emocional de los demás y del propio niño. De la misma forma, el proceso de formación de las acciones simbólicas en los niveles materializado, perceptivo y verbal durante el juego de roles sociales posibilitó que los niños organizaran su actividad, de manera que se garantizó que pudieran considerar objetivos compartidos conscientemente al representar los roles, lo cual tiene que ver con la actividad voluntaria así como con la capacidad para comportarse independientemente de las circunstancias que influyen directamente sobre ellos. En palabras de Bozhovich (1978, p. 151) "el surgimiento de esta capacidad condiciona el carácter activo y no reactivo de la conducta del niño”.

Del mismo modo, en esta investigación se encontró que la orientación que el adulto brindó en el juego posibilitó que los niños desarrollaran habilidades específicas en cada uno de los niveles de la función simbólica (acciones materializadas, acciones perceptivas y acciones verbales) y esto a su vez permitió que desarrollaran la iniciativa en cada nivel de las acciones simbólicas y luego que las transformaran creativamente en correspondencia con las necesidades de las temáticas del juego y de los roles que representaban. Esto significa que la orientación sistemática en el juego posibilitó que los niños aprendieran a observar diversas situaciones en el juego como las normas morales y que adquirieran rasgos cualitativamente nuevos tanto de esta actividad como de la función simbólica, lo que se expresó en el desarrollo de características nuevas de su personalidad al cumplir con deberes ante sus compañeros de juego, al colaborar con ellos y con el adulto, al contar no solo con sus intereses personales sino también con las necesidades de los otros niños, al adquirir un nuevo sentido de las relaciones sociales transformandose 
en simpatía por los otros, en sentimientos de identificación con las alegrías y tristezas ajenas como si fueran suyas así como lo plantea Zaporozherts (1978).

Igualmente, de acuerdo a los hallazgos de este estudio se puede decir que la experiencia de juego de roles sociales con el uso de medios simbólicos permitió que los niños comprendieran sus propias características, las de los demás y el entorno, y esto permitió una participación e implicación emocional más profunda en la vida social (ver figura 6), esta afirmación coincide con investigaciones previas realizadas (GONZÁLEZMORENO, 2016). Esto significa que las formas maduras del juego de roles sociales y del desarrollo complejo de la función simbólica con el uso del lenguaje simbólico permiten el desarrollo de la personalidad al posibilitar el encuentro con las experiencias del otro para identificar sus estados emocionales y así transformar las propias acciones. Esto se expresa en que los niños empezaron a ser más responsables de sus propias acciones porque desarrollaron habilidades de autorregulación emocional y de sus actitudes, así como dominio del propio comportamiento en las interacciones comunicativas dialógicas.

Figura 6 - Relación juego de roles sociales, función simbólica y personalidad

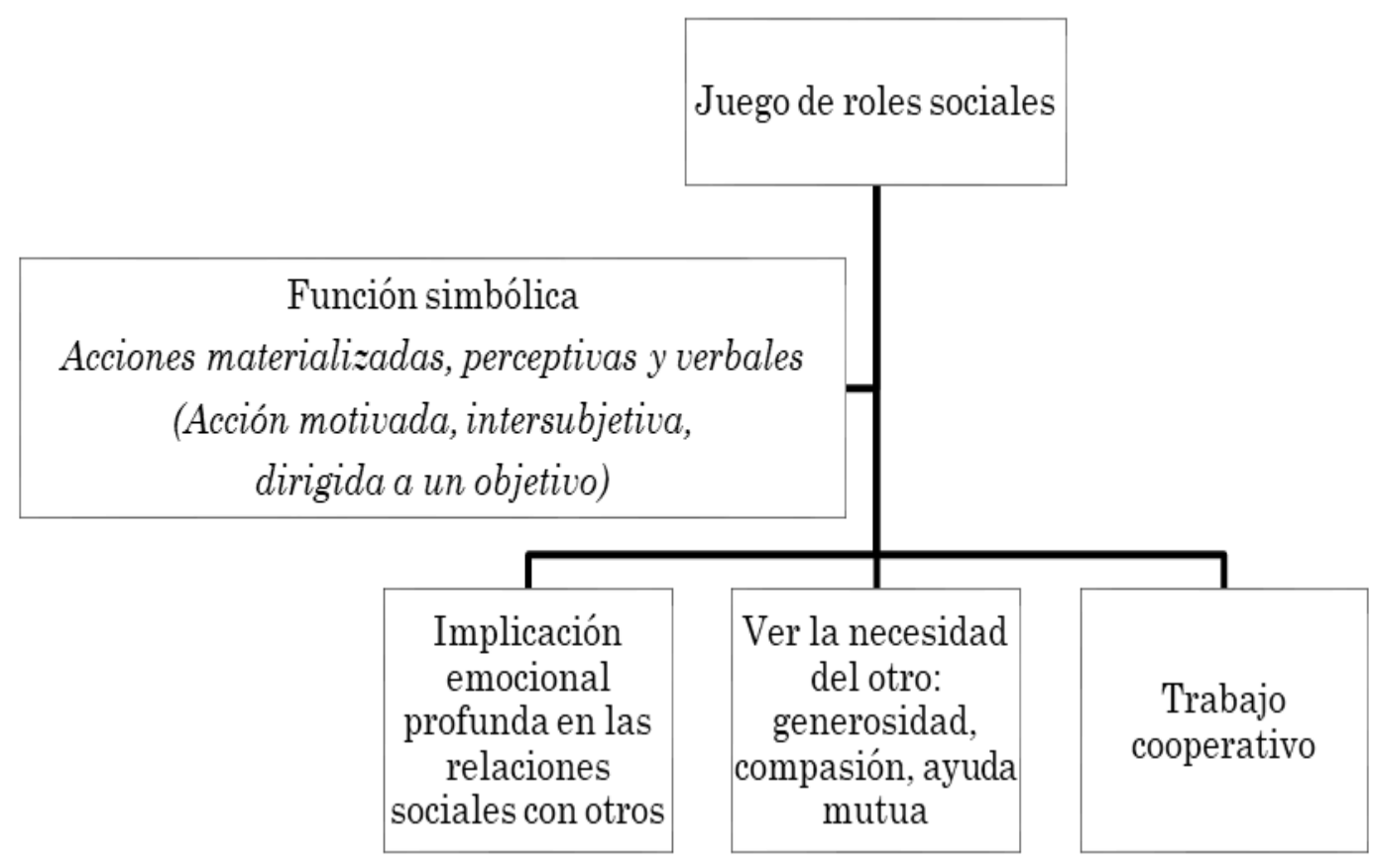

Fuente: Elaboración propia 
Por lo anterior, se reflexiona respecto a la necesidad de considerar los tipos de acciones que se puedan realizar en las diversas etapas formativas tanto del juego de roles sociales como de la función simbólica para el desarrollo de la personalidade en la edad preescolar. La función simbólica como proceso psicológico experimenta complejas transformaciones en el juego de roles sociales, surge a partir de la participación colectiva de los niños en esta actividad. Cuando el juego de roles se transforma hacia las formas maduras, la función simbólica contribuye con la transformación del sentido mismo de las interacciones sociales. En este proceso la zona del desarrollo próximo también se transforma en la zona del desarrollo actual del desarrollo psicológico del niño. Esto requiere de la identificación por parte del adulto de las necesidades del grupo de niños para que pueda proporcionar los apoyos que posibiliten que el juego avance y el desarrollo psicológico del niño también.

De igual manera, se reflexiona respecto a considerar la dialéctica del desarrollo psíquico del niño, en cuyo curso, se formaron nuevas estructuras psíquicas cualitativamente específicas durante la asimilación de la experiencia social en el juego, lo cual contribuyó a formar: cualidades morales de la personalidad, es decir, normas morales de conducta no bajo la influencia de coacciones externas sino por iniciativa al pensar en el bien común de los compañeros de juego de roles sociales; los sentimentos morales y estéticos, así como los motivos sociales de comportamento. Esto posibilitó el cambio del carácter de las vivencias emocionales de los niños, lo que los movió a pasar a la actividad más compleja y más significativa socialmente, así como lo plantea Zaporozhets (1987), que en este caso se refiere al juego de roles narrativo.

Esta investigación está en sintonía con estudios realizados por Leontiev (1987) en los que se afirma que durante la participación del niño en el juego hacia sus etapas más maduras, el pequeño desarrolla un sistema de motivos jerarquicamente subordinados que comienza a guiar su comportamiento y a determinar su desarrollo. Aunque al inicio mismo del juego no se presenta de esta manera porque el niño se deja llevar por estímulos e impulsos instantáneos que actúan sobre él. Despué el niño es capaz de guiarse por 
aspiraciones y deseos estables, ligados a la asimilación de normas sociales de vida (BOZHOVICH, 1978, 1987).

Asimismo, se reflexiona respecto a que en el juego de roles las interaciones sociales de los niños se transforman entre ellos mismos y con el adulto que los acompaña. Esto asi como lo propone Elkonin (1980) posibilita la creación de una situación social especial en la que los pequeños asimilan normas sociales de comportamiento, satisfacen varias necesidades de vínculo con el otro, se enriquecen las emociones y surgen formas complejas de la actividad, de acciones e interrelaciones. Estas particularidades psicológicas en los niños les permitieron el desarrollo de la capacidad de guiarse por instancias éticas. Por esta razón, se afirma que el juego de roles sociales cumple con una función especial en la formación moral de los niños al permitirles representar roles y distinguir en ellos normas y reglas que son aceptadas en el medio social que los rodea y las hacen normas de su comportamiento en el juego (BOZHOVICH, 1987). Por ejemplo, los niños sienten alegría y satisfacción personal cuando se dan cuenta que cumplieron su rol en el juego y que ayudaron a alguien en problemas, lo cual está estrechamente vinculado con sus vivencias emocionales. Estos sentimientos se constituyen en la base de los sentimientos morales que se forjaran en las siguientes edades del desarrollo psicológico del niño. En palabras de Bozhovich (1987, p. 268) "estas instancias morales en la edad preescolar generan los motivos morales del comortamiento".

Se concluye que el juego de roles sociales se constituye en una actividad en la que los medios simbólicos usados contribuyen a que se establezcan vínculos afectivos entre los participantes, se genere la atención activa hacia las interacciones con los otros y se forjen actitudes compasivas y bondadosas hacia los demás. Asimismo, el niño adquiere, interioriza y enriquece la experiencia emocional e interaccional durante su participación en la actividad de juego de roles sociales con el uso de medios simbólicos.

Otra conclusión se refiere a que el éxito de la formación de la función simbólica en los niveles materizalizado, perceptivo y verbal como acciones nuevas 
depende del tipo de orientación que la investigadora proporcionó para descubrir ante los niños el contenido propio de la acción con la que participaban en el juego de roles. Cada acción era desplegada y exteriorizada ante el grupo de niños al inicio para reducirla gradualmente hasta su interiorización.

Asimismo, los hallazgos de este estudio posibilitan suponer que los cambios ocurridos durante las acciones simbólicas (materializadas, perceptivas y verbales) crean las premisas para la formación de la personalidad en la edad preescolar sobre la base de la reestructuración de la orientación durante la consecusión de las etapas de juego de roles sociales. Por ello, este trabajo es evidencia novedosa sobre la manera en la que el juego de roles en sus formas maduras contribuye con el desarrollo complejo de la función simbólica y esto a su vez con el desarrollo de la personalidad infantil en la edad preescolar.

Finalmente, los hallazgos obtenidos en esta investigación y en otras realizadas (GONZÁLEZ-MORENO; SOLOVIEVA; QUINTANAR, 2014; GONZÁLEZ-MORENO, 2015， 2016, 2018) permiten corroborar la importancia de propiciar las condiciones óptimas en la edad preescolar para el desarrollo potencial y armónico de los niños, el cual se da durante su participación en el juego de roles sociales con el uso de medios simbólicos lo que tiene un impacto importante en el desarrollo de la personalidad, en lugar de transformar prematuramente la edad preescolar en la escolar con la introducción forzada de letras y números. Así como lo plantea Zaporozhets (1978) en la edad preescolar es necesario el despliegue amplio y el enriquecimiento máximo del contenido del juego de roles sociales y de la comunicación dialógica de los participantes. Cuando el niño en edad preescolar no tiene la posibilidad de participar en actividades de juego de roles sociales y es forzado a participar en actividades que corresponden a la edad escolar presenta con frecuencia dificultades en el desarrollo de la motivación para aprender, en las interacciones con los demás, en la gestión de sus emociones, en el desarrollo de los aspectos morales, en su comportamiento y en su esfera emocional-afectiva (GONZÁLEZ-MORENO; 
SOLOVIEVA, 2016). Además, si el niño en edad preescolar no tiene el tiempo suficiente y necesario para participar de manera frecuente en diferentes roles sociales en el juego, esta actividad no madura ni se complejizan las formaciones psicologicas que se espera que se desarrollen en este momento especial de la vida (e.g. la función simbólica, el pensamiento reflexivo, la actividad voluntaria) y esto a su vez tiene efectos negativos en el desarrollo de su personalidad así como lo señalan diversos estudios (HAKKARAINEN; BREDIKYTE, 2015; GONZÁLEZ-MORENO, 2015, 2016, 2018 ).

\section{Referencias}

ELKONIN, D. B. Psicología del Juego. Madrid: Visor Libros, 1980.

BOZHOVICH, L. Las etapas de la formación de la personalidad en la ontogénesis. EN: I.I. Iliasov \& V. Y. Liaudis (Eds.), Antología de la psicología pedagógica y de las edades. La Habana: Pueblo y Educación. p.151-161, 1978.

BOZHOVICH, L. Las etapas de formación de la personalidad en la ontogenesis. EN: En M. Shuare y V. Davídov (compiladores). La psicologia evolutiva y pedagógica en la URSS. Moscú: Editorial Progreso. p. 250-274, 1987.

BREDIKYTE, M.; HAKKARAINEN, P. Play intervention and play development. IN: Lobman C, O’Neill B. editors. Play and performance. Lanham: University Press of America. p. 59-83, 2011.

GALPERIN, P. YA. Introducción a la psicología general. Moscú: Universidad Estatal de Moscú, 1976.

GALPERIN, P. YA. El método de cortes y el método de formación por etapas en el estudio del pensamiento infantil. IN: BURMENSKAYA, G. V. (ED.). Compilación sobre psicología infantil. Moscú: Instituto de Psicología Práctica, 1995.

GALPERIN, P. YA. Cuatro conferencias sobre psicología. Moscú: Escuela Superior, 2000.

GONZÁLEZ-MORENO, C.X. Formación de la función simbólica por medio del juego temático de roles sociales en niños preescolares. Rev. Fac. Med. v. 63, n 2, p. 235-41, 2015. DOI: http://dx.doi.org/10.15446/revfacmed.v63n2.47983.

GONZÁLEZ-MORENO, C.X. El juego de roles sociales por etapas para promover la formación de la función simbólica por niveles de desarrollo en niños preescolares. Tipica, Boletín Electrónico de Salud Escolar. v. 12, n. 2, p. 78-93, 2016. 
GONZÁLEZ-MORENO, C.X. Actividad comunicativa interactiva en una experiencia de juego grupal con niños en edad preescolar, Cuadernos Hispanoamericanos de Psicología. v. 17, n. 2, p. 43-60, 2018.

GONZÁLEZ-MORENO, C. X.; SOLOVIEVA, Y.; QUINTANAR, R. L. El juego temático de roles sociales: aportes al desarrollo en la edad preescolar. Avances en Psicología Latinoamericana, v. 32, n. 2, p. 287-308, 2014. DOI: http://dx.doi.org/10.12804/apl32.2.2014.08.

GONZÁLEZ-MORENO, C.X.; SOLOVIEVA, Y. Impacto del juego de roles sociales en la formación de la función simbólica en preescolares. Revista de Psicología Universidad de Antioquia, v. 8, n. 2, p. 49-70, 2016. DOI:

http://dx.doi.org/10.17533/udea.rpsua.v8n2a04.

HAKKARAINEN, P.; BREDIKYTE, M. How play creates the zone of proximal development. IN: ROBSON, S; FLANNERY, S. (ED.). The Routledge International Handbook of Young Children's Thinking and Understanding. New York: Routledge. p. 31-42, 2015.

LEONTIEV, A. El desarrollo psíquico del niño en la edad preescolar. EN: En M. Shuare y V. Davídov (compiladores). La psicologia evolutiva y pedagógica en la URSS. Moscú: Editorial Progreso. p. 57-70, 1987.

SOLOVIEVA, Y.; QUINTANAR, L. La actividad de juego en la edad preescolar. México: Trillas, 2012.

SOLOVIEVA, Y. La actividad intelectual en el paradigma histórico-cultural. México: Ediciones CEIDE; 2014.

TALIZINA, N. F. La teoría de la actividad aplicada a la enseñanza. México: Universidad Autónoma de Puebla, 2019.

VIGOTSKY, L. S. Obras escogidas. Tomo III. Madrid: Visor, 1995.

ZAPOROZHETS, A. La importancia de los periodos tempranos de la infancia para la formación de la personalidad infantil. EN: I.I. Iliasov \& V. Y. Liaudis (Eds.), Antología de la psicología pedagógica y de las edades. La Habana: Pueblo y Educación. p. 148-151, 1978.

ZAPOROZHETS, A. Importancia de los periodos iniciales de la vida en la formación de la personalidad infantil. EN: En M. Shuare y V. Davídov (compiladores). La psicologia evolutiva y pedagógica en la URSS. Moscú: Editorial Progreso. p. 228-249,1987. 